\title{
Pseudospark Experiments: Cherenkov Interaction and Electron Beam Post-Acceleration
}

\author{
H. Yin, A. W. Cross, W. He, A. D. R. Phelps, and K. Ronald
}

\begin{abstract}
Pseudospark (PS) discharge experiments to generate high-brightness electron beams have been carried out at the Strathclyde University, Glasgow, U.K. The PS-sourced electron beam has two phases, an initial 22-kV, 50-A hollow-cathode phase (HCP) beam of brightness $10^{9-10} \mathrm{Am}^{-2} \mathrm{rad}^{-2}$ followed by a 200-V, 200-A conductive phase $(C P)$ beam of brightness $10^{11-12}$ $\mathrm{Am}^{-2} \mathrm{rad}^{-2}$. The initial HCP beam from an eight-gap PS discharge was applied for the first time in a Cherenkov interaction between the electron beam and the $T M_{01}$ mode of a $60-\mathrm{cm}$ long alumina-lined waveguide. A gain of $29 \pm 3 \mathrm{~dB}$ was measured and an output power of $2 \pm 0.2 \mathrm{~kW}$ in the frequency range 25.5-28.6 GHz. Another experiment was focused on the study of the propagation and post-acceleration of the $\mathrm{CP}$ beam from a three-gap PS discharge chamber. The beam was successfully accelerated from about $200 \mathrm{~V}$ to more than $40 \mathrm{kV}$.
\end{abstract}

Index Terms-Cherenkov interaction, electron beams, post-acceleration, pseudospark (PS).

\section{INTRODUCTION}

$\mathbf{P}$ SEUDOSPARK (PS) discharge [1]-[3] experiments to generate a low-temperature plasma for use as a copious source of electrons have been carried out at the University of Strathclyde, Glasgow, U.K. [4], [5]. The plasma can be regarded as a low-work function surface that facilitates electron extraction. Electron beam pulses of duration of tens of nanoseconds, current density $\left(>10^{8} \mathrm{Am}^{-2}\right)$, brightness of up to $10^{12} \mathrm{Am}^{-2} \mathrm{rad}^{-2}$ and emittance of tens of millimeters mrad were measured from a PS discharge. This beam has a higher combined current density and brightness compared to electron beams formed from any other known type of electron source. A PS-based, high-intensity, high-brightness electron beam source was applied for the first time in a free-electron maser device at the University of Strathclyde [6], [7].

Another experiment was focused on the study of the propagation and post acceleration of the beam from a three-gap PS discharge chamber. A beam from the PS discharge was transported in a plasma induced ion background and simultaneously accelerated by an accelerating potential. This makes it possible to provide a higher energy electron beam with lower energy spread for future application in a high-power free-electron maser. This paper will present some results from both the Cherenkov interaction and post-acceleration experiments.

\section{EXPERIMENTAL SETUP}

\section{A. Cherenkov Maser Experimental Setup}

A schematic outline of the PS-based Cherenkov maser amplifier is shown in Fig. 1. The main components of the experiment are the PS-based electron beam source, the magnetic field for beam transport, the Cherenkov interaction region, electrical/beam diagnostics, and the microwave launching/diagnostic system. A more detailed description of the experimental setup can be found in [7].

In the maser system, the presence of the dielectric in the waveguide reduces the phase velocity of the electromagnetic waves, allowing a resonant interaction to occur between a TM or HE waveguide mode and the rectilinear electron beam. Coherence of the generated radiation arises due to bunching of electrons in phase with respect to the electromagnetic wave. For values of the beam and waveguide parameters relevant to this experiment, the force exerted on the electrons by the waveguide mode was dominated by the resonant space-charge force, so the maser operated in a Raman-type regime, with strongest amplification of the waveguide mode expected when it was resonant with the slow space-charge wave of the beam, i.e., when the angular frequency $\omega$ and the axial wavenumber $k_{z}$ of the waveguide mode satisfy

$$
\omega \approx k_{z} v_{z}-\frac{\omega_{p}}{\gamma}
$$

where $v_{z}$ is the axial electron velocity, $\gamma=\left[1-(v / c)^{2}\right]^{-1 / 2}=$ $\left[1-\beta^{2}\right]^{-1 / 2}$ is the relativistic factor, $\omega_{p}=\sqrt{e^{2} n_{e} / \varepsilon_{0} \gamma m_{0}}$ is the plasma frequency, $n_{e}$ is the electron density, $\beta=v / c$, is the relative velocity, $v$ is the total electron velocity, $c$ is the speed of light, and $e$ and $m_{0}$ are the electronic charge and rest mass, respectively. $\omega$ and $k_{z}$ must also, of course, satisfy the characteristic equation for a dielectric-lined cylindrical waveguide, which for a $\mathrm{TM}_{0 n}$ mode is as in [7]. From the space-charge mode of the electron beam and the $\mathrm{TM}_{01}$ and $\mathrm{TM}_{02}$ waveguide modes for this Cherenkov maser experiment, a resonant interaction of the beam mode would be expected to occur around $21 \mathrm{GHz}$ with the $\mathrm{TM}_{01}$ mode and $55 \mathrm{GHz}$ with the $\mathrm{TM}_{02}$ mode.

The vacuum pressure and gas pressure were measured 110 $\mathrm{cm}$ away from the anode near the vacuum pump port and at the anode, respectively, by two active Pirani gauge heads and recorded on a two channel digital display. The charging voltage was measured by an instantaneous capacitive voltage probe. The discharge current was monitored by an in-line current viewing resistor (CVR) of resistance $0.066 \Omega$. The electron-beam parameters measured included the beam current 


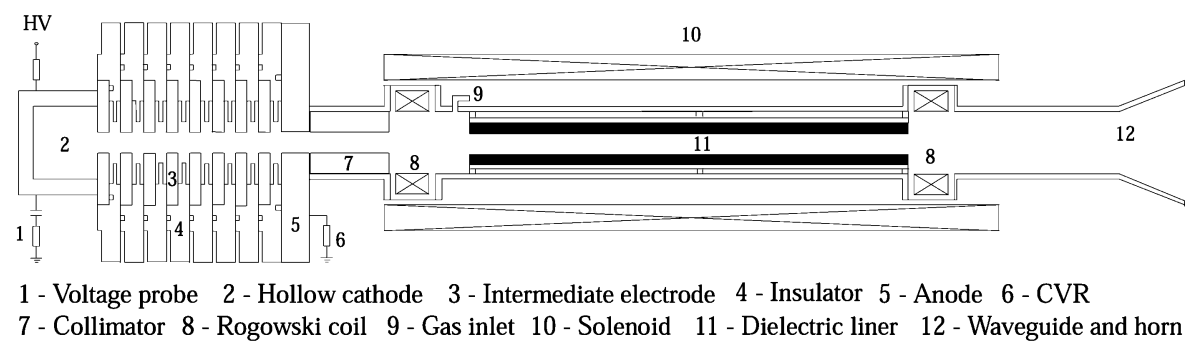

Fig. 1. Cherenkov maser experimental configuration.

(using a compact Rogowski coil upstream from the interaction region) and the transported beam current (using another compact Rogowski coil downstream from the interaction region). The microwave pulse duration and temporal profile from the PS-based Cherenkov maser amplifier were obtained by monitoring the output of a rectifying crystal detector with an oscilloscope. The basic microwave measurement system, screened in a metal box, was composed of Ka-band (26.5-40 $\mathrm{GHz})$ or W-band $(75-100 \mathrm{GHz})$ components starting with a small microwave receiving horn followed by a directional coupler, attenuators, waveguide, and crystal detectors. Two methods were used to identify the frequency range of the Cherenkov radiation. One technique used a series of in-waveguide cutoff filters, and the other used an interferometer. From the first method, both the frequency and the energy distribution were obtained.

The radiation mode pattern (i.e., the field distribution and the power distribution) measurements of the Cherenkov maser were conducted by scanning a radially polarized detector in a circular path centred on the aperture of the output horn while repeatedly pulsing the maser device. The field distribution and the power distribution are dependent on the distance from the launching antenna to the detector, which can be divided into three identifiable regions without sharp boundaries including the reactive near-field region (Rayleigh field), the radiating near-field region (Fresnel region), and the far-field region (Fraunhofer region). The power distribution measurements were conducted in the far-field region. For the Cherenkov maser, the diameter of the output horn was $6 \mathrm{~cm}$ and the dominant frequency of the source was about $25.5 \mathrm{GHz}(\lambda=1.18 \mathrm{~cm})$ and the far field conditions required that the launching and receiving antenna should be separated by larger than $61 \mathrm{~cm}$. Therefore, the detector was placed at $1 \mathrm{~m}$ away from the launching horn. A reference detector system was detached and placed at a fixed position through the entire mode scanning experiment to compensate for random fluctuations in the maser output.

\section{B. Electron Beam Post-Acceleration Experimental Setup}

A schematic outline of the experimental setup for the study of the propagation and the post-acceleration of the PS-sourced beam is shown in Fig. 2. The electron beam was extracted from a three-gap PS discharge chamber. The discharge chamber consisted of a planar anode, a planar cathode with a cylindrical hollow cavity, two intermediate electrodes of 3.25 -mm thickness and three Perspex insulators of 3.25-mm thickness. The cylindrical hollow cathode cavity was made of stainless steel having outer and inner diameters of 63 and $50 \mathrm{~mm}$, respectively,

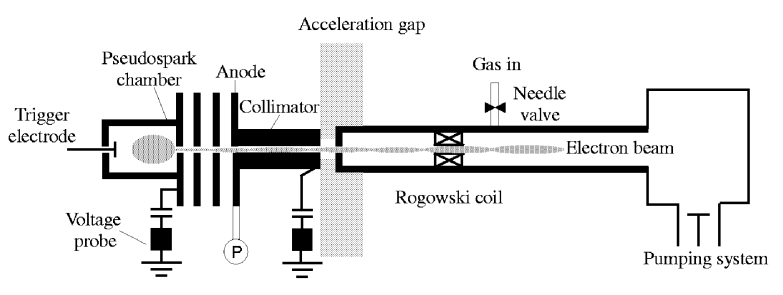

Fig. 2. Schematic diagram for the post-acceleration experiment of the PS electron beam.

and length $50 \mathrm{~mm}$, in which a trigger electrode made of coaxial cable was inserted. Both the anode and cathode had an on-axis hole of 3-mm diameter. The post-acceleration gap was located $30 \mathrm{~mm}$ away from the anode of the PS discharge chamber. The single Perspex disc of inner diameter $5 \mathrm{~mm}$, outer diameter 300 $\mathrm{mm}$, and 26-mm thickness was used for acceleration gap insulation. It was made with a recess to achieve a $5-\mathrm{mm}$ acceleration gap separation.

A Rogowski coil was located $120 \mathrm{~mm}$ downstream of the post-acceleration gap to measure the beam current. The gas inlet was located $30 \mathrm{~mm}$ away from the Rogowski coil and was controlled by a very fine mechanical needle valve. The background vacuum requirements were obtained using a two stage pumping system in the form of a rotary pump and an oil-diffusion pump. Air was used throughout the experiments. The pressure was measured by a Baratron type vacuum gauge together with a display meter.

The PS discharge was powered by a dc power supply. The hollow cathode was connected through a $30-\mathrm{M} \Omega$ charging resistor to a negative voltage source $(-30 \mathrm{kV}, 1-\mathrm{mA}$ dc power supply) and the charging voltage was measured by a capacitive voltage probe. The external energy storage capacitance 600 $\mathrm{pF}$ was achieved by using three chains of five $1000-\mathrm{pF}, 15-\mathrm{kV}$ resin-dipped ceramic capacitors in series across the cathode and anode of the PS discharge chamber. The acceleration unit was driven by a $40-\mathrm{kV}, 125-\mathrm{ns}$ voltage pulse produced by a cable Blumlein and the acceleration voltage was measured by another capacitive voltage probe. The cable Blumlein was made by winding in parallel several $50-\Omega$ coaxial cables of $25-\mathrm{m}$ lengths around a spindle to form a Blumlein type of pulse generator. The internal impedance of the cable Blumlein was reduced by increasing the number of the cables used. The PS discharge and the cable Blumlein were triggered by two sets of trigger signals controlled by two delay units and one trigger source. Both trigger signals for the PS and the cable Blumlein were $15-\mathrm{kV}$ pulses. Careful adjustment of the delay units ensured the beam acceleration voltage was applied at the right time. 


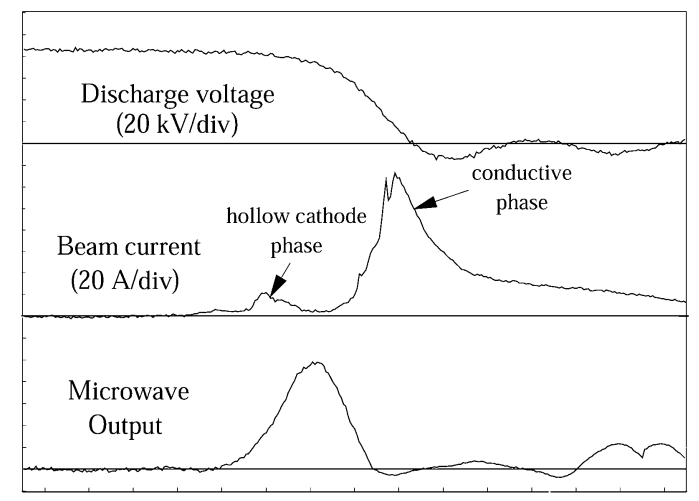

Time (20ns/div)

Fig. 3. Typical waveforms of PS discharge voltage, the beam current, and the microwave pulse.

\section{EXPERIMENTAL RESULTS}

\section{A. Cherenkov Maser Experimental Results}

The operation of the whole maser system is described as follows. The maser system was originally evacuated down to 2-3 mtorr at the discharge chamber and $\sim 0.01$ mtorr at the pumping port. The pumping port was connected at the end of the output horn using a glass "T-piece" chamber. Argon gas was fed into the system from the anode side at a very slow rate of $\sim 1$ mtorr/s through a very fine controlled needle valve until a desired gas pressure was reached and balanced by adjustment of the needle valve. The high voltage applied across the PS chamber was increased slowly until breakdown occurred. To achieve a self-pinched, high-current electron beam, it was essential to match the applied discharge voltage and chamber gas pressure so that the discharge operated in the PS regime. Our experiments showed an empirical relation of $v_{B}=(0.20 \pm 0.05) \times p^{-4.02 \pm 0.18} d^{-1.77 \pm 0.01}$, where $V_{B}$ is the breakdown voltage of the PS discharge in kilovolts, and $p$ and $d$ are the pressure in torr and the cathode-anode separation in centimeters, respectively. The self-pinched electron beam was extracted from the anode hole of the eight-gap PS discharge chamber just before breakdown occurred and then guided along the beam tube to the Cherenkov interaction region, where the beam interacted with the modes of the dielectric lined waveguide, and the resonant wave was amplified to a few kilowatts from a low power level of a few watts. The microwave radiation generated in the interaction region was guided a further $60 \mathrm{~cm}$ in a cylindrical vacuum waveguide before being launched into free space by the conical output horn. The microwave radiation was then diagnosed by a screened and calibrated microwave diagnostic system situated in the far field of the antenna.

Microwave radiation was detected successfully from the PS-based dielectric Cherenkov maser amplifier. The temporal profile of the microwave output radiation from the maser is shown in the lower part of Fig. 3, while time correlated with the electron-beam current and voltage profiles. It was found experimentally that significant microwave radiation was generated only when the dielectric was present in the interaction space, although if there was no dielectric in the cylindrical waveguide, then a very small background microwave output was detected.

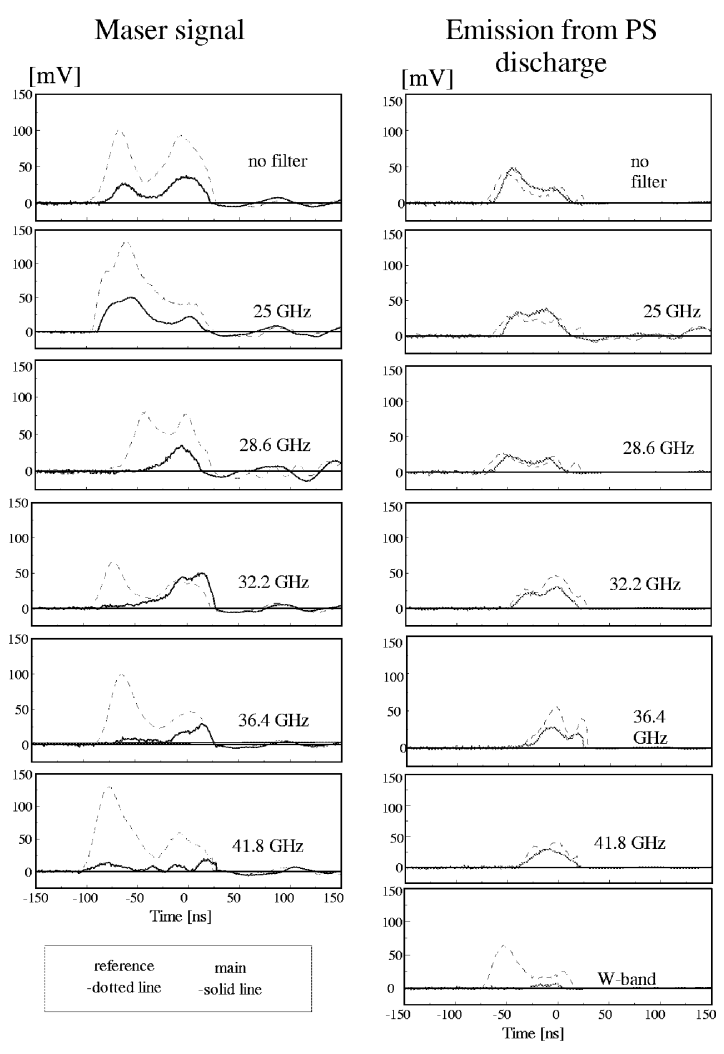

Fig. 4. Results with cutoff filters for the emissions from the PS and the Cherenkov maser output signal.

This demonstrated in conjunction with the observation that the microwave output signal was independent of the guide magnetic field over the range $0.13-0.26 \mathrm{~T}$, that the radiation from the experiment was due to the Cherenkov interaction mechanism. In addition, two components of the microwave pulse were observed corresponding to the two energy components of the electron beam during the PS discharge breakdown. Another interesting result was the discovery that the small background signal was always present even without the guide B-field or dielectric lining in the waveguide. These results demonstrated that the microwave radiation grew from the background seed signal, which seemed to be from the PS discharge itself [9], [10].

The frequency range of the microwave radiation from the Cherenkov maser amplifier was measured by applying different cylindrical cutoff filters in the waveguide and comparing this signal with a signal from a nonfiltered reference detector. Using this method, both the Cherenkov microwave output and the background signal with no dielectric liner present were analyzed. Fig. 4 shows the microwave output results with cutoff filters for both the background signal and the Cherenkov maser output signal when the applied discharge voltage was fixed at $\sim 75 \mathrm{kV}$. The waveforms in Fig. 4 were acquired by a digitizing oscilloscope in an averaging mode of five shots. The "background signal" means the output signal without the dielectric liner in the waveguide. The results showed that the signal in the absence of the dielectric, i.e., PS discharge radiation, had a wide frequency distribution but below the W-band (62.5 $\mathrm{GHz}$ ) cutoff frequency. In the presence of the dielectric, the 


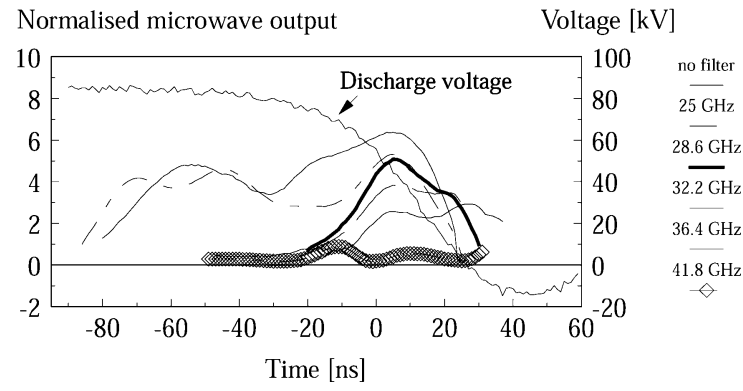

Fig. 5. Normalized Cherenkov maser output signals with various high-pass filters and the time-correlated accelerating potential.

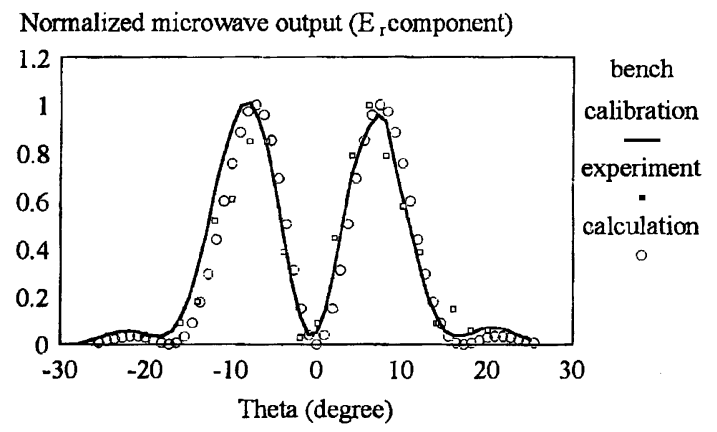

Fig. 6. Far-field mode pattern scan of the radial component $E_{r}$ compared with calculations and bench calibration.

microwave output had typically two frequency components: one was between 25.5 and $28.6 \mathrm{GHz}$ and the other between 28.6 and $41.8 \mathrm{GHz}$. By normalizing the Cherenkov maser output signals in Fig. 4, an averaged, normalized microwave output was obtained for each cut-off filter configuration and is shown in Fig. 5. By comparing the time evolution of the beam voltage with the averaged microwave output spectrum, it was found that the low-frequency microwave signal corresponded to a $70-$ to $80-\mathrm{keV}$ beam energy from the hollow-cathode discharge regime and the higher frequency microwave signal to the lower energy beam from the conductive phase of the PS discharge. This increase in frequency with decreasing beam energy is consistent with a Cherenkov interaction mechanism when the phase velocity of the wave mode is larger than its group velocity ( $\left.V_{\text {phase }}>V_{\text {group }}\right)$, as in our case.

To verify the mode of operation as $\mathrm{TM}_{01}$, the far-field output radiation pattern from the conical output horn, which had an aperture diameter of $60 \mathrm{~mm}$, was measured. The output antenna pattern associated with the azimuthal E-field component was measured to be independent of the presence of the dielectric and close to zero, confirming the operation of a TM mode. Fig. 6 shows the measured pattern associated with the radial E-field component of the radiation with a relative error of $4 \%$ and a systematic error in the radial angle measurement of $<2^{0}$. The measured pattern was in good agreement with the results from bench experiments in which a $27-\mathrm{GHz} \mathrm{TM}_{01}$ microwave signal was launched using the same horn. Fraunhofer diffraction theory predicts a maximum at $7.5^{\circ}$, which is in close agreement with both the Cherenkov maser experiment and bench measurements.

In order to measure the absolute power of the microwave pulse from the Cherenkov maser, the microwave energy received by a $15-\mathrm{dB}$ gain rectangular receiving horn with
TABLE I

Propagation of THE PS BEAM THROUgh a COLLIMATOR

Beam measured at $150 \mathrm{~mm}$ away

from the PS anode

Collimator

Beam current/A Percentage of

length $/ \mathrm{mm}$

beam

transported

\begin{tabular}{ccc} 
No collimator & $240 \pm 35$ & $\cdots$ \\
30 & $168 \pm 20$ & $70 \% \pm 2 \%$ \\
60 & $118 \pm 10$ & $49 \% \pm 3 \%$ \\
90 & $36 \pm 5$ & $15 \% \pm 0.1 \%$ \\
\hline \hline
\end{tabular}

an effective area of $3.5 \mathrm{~cm}^{2}$ (for the microwave frequency of $25.5 \mathrm{GHz}$ ) was fed to a well-calibrated crystal detector. The power density at a range of $1 \mathrm{~m}$ and at the maximum of the antenna pattern was measured to be $1.6 \pm 0.2 \mathrm{Wcm}^{-2}$. Therefore, the maximum total power from the Cherenkov maser was calculated to be $2.0 \pm 0.2 \mathrm{~kW}$ (without considering any losses within the detecting system) by integrating the measured normalized mode pattern. After similar treatment to the background microwave seed signal from the PS itself without maser amplification, the background microwave seed signal was found to be $2.7 \mathrm{~W}$ in the frequency range between 25.5-28.6 GHz. This corresponded to a gain of $29 \pm 3 \mathrm{~dB}$.

The observed frequency was found to be $20 \%$ higher than that predicted by the resonance condition for 70 - to $80-\mathrm{keV}$ beam energy. This discrepancy is probably due to charging of the dielectric liner [11]. A relative spectral energy distribution was obtained and approximately $65 \%$ of the radiation was found to lie in the 25- to $28.6-\mathrm{GHz}$ frequency band.

\section{B. Post-Acceleration Experiments}

The propagation of the electron beam from a three-gap PS discharge chamber was studied as a function of the length of a collimator of $3.5-\mathrm{mm}$ internal diameter. In this experiment, the post-acceleration unit was initially disconnected, and only the three-gap PS discharge chamber was used. The chamber was first evacuated through a small hole in the PS anode by the pumping system typically down to a pressure of 3 mTorr. The desired gas pressure for the PS was reached and balanced by adjusting a very fine mechanical needle valve. The high voltage applied across the PS chamber was then increased slowly until breakdown occurred. To achieve a high-current electron beam, it was essential to match the applied discharge voltage and gas pressure in the chamber so that the discharge operated in the PS regime [8]. The beam was measured by a Rogowski coil positioned $150 \mathrm{~mm}$ away from the anode of the PS chamber. Rogowski coil beam current measurements $150 \mathrm{~mm}$ downstream from the anode showed that with no magnetic guiding field, $70 \%$ and $50 \%$ of the beam propagated through 30 - and $60-\mathrm{mm}$ long collimators, respectively, as shown in Table I. 


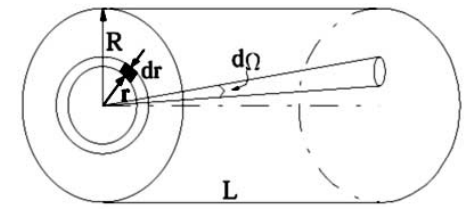

Fig. 7. Configuration of the field-free collimator for the brightness measurement on the PS e-beam source.

Beam normalized brightness [A m $\left.{ }^{-2} \mathrm{rad}^{-2}\right]$

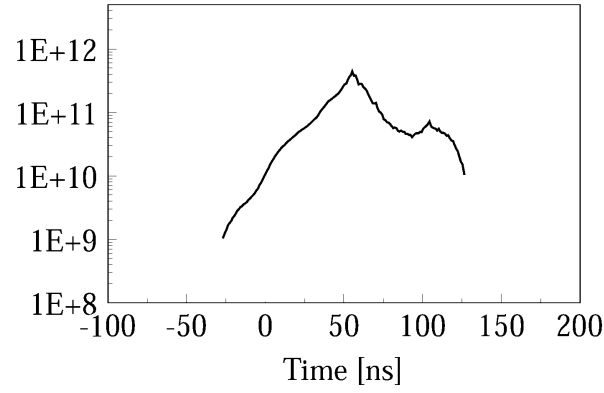

Fig. 8. Measured beam brightness from a three-gap PS discharge.

A magnetic-field free-collimator technique enabled a beam brightness of up to $10^{11-12} \mathrm{Am}^{-2} \mathrm{rad}^{-2}$ to be measured from a three-gap PS discharge. The principle of this method is described in Fig. 7. A cylindrical collimator of radius $R$ and length $L$ in a magnetic field-free condition is installed concentrically with the electron beam. The beam electrons move divergently in straight lines and the electrons with larger transverse velocity are collimated if $R / L$ is carefully selected such that

$$
R / L \leq x_{\max }^{\prime}
$$

where $x_{\max }^{\prime}$ is the maximum transverse angle of the electrons. The four-dimensional (4-D) trace space area of the collimated electron beam can be calculated as

$$
v_{4}=\iiint d x d x^{\prime} d y d y^{\prime}=\iint d s d \Omega
$$

where $d s=2 \pi r d r, \int d \Omega=\pi R^{2} / L^{2}$. Therefore, the brightness of the collimated electron beam is given by

$$
B_{n}=\frac{2 I L^{2}}{\beta^{2} \gamma^{2} \pi^{2} R^{4}} .
$$

Here, $\beta=v / c$ is the relative velocity, $v$ the total electron velocity, and $c$ the speed of light. $\gamma=\left[1-(v / c)^{2}\right]^{-1 / 2}=$ $\left[1-\beta^{2}\right]^{-1 / 2}$ is the relativistic factor. The setup of the beam brightness measurement on the PS experiment is also illustrated in Fig. 2. The brightness $B_{n}$ can be measured by measuring the beam current $I$ and the corresponding voltage which determine the values of $\beta$ and $\gamma$, while $L$ and $R$ in our experiment were 60 and $1.75 \mathrm{~mm}$, respectively. Fig. 8 shows a measured time-resolved normalized brightness of the electron beam from the three-gap PS. At a discharge voltage of $20 \mathrm{kV}$, the measured normalized brightness of the $\mathrm{CP}$ beam was found to be in the order of $10^{11} \mathrm{Am}^{-2} \mathrm{rad}^{-2}$.

In the post-acceleration experiment a 30-mm-long collimator of $3.5-\mathrm{mm}$ internal diameter was used after the anode of the PS

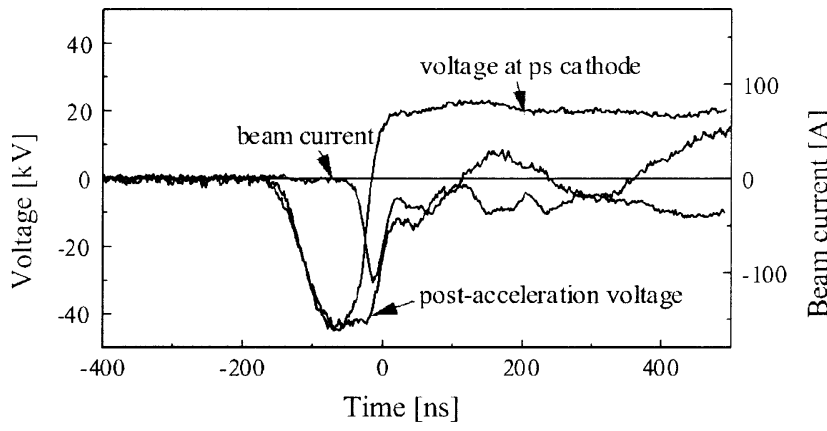

Fig. 9. Typical record of the time-correlated PS discharge voltage, beam current, and the acceleration voltage pulse.

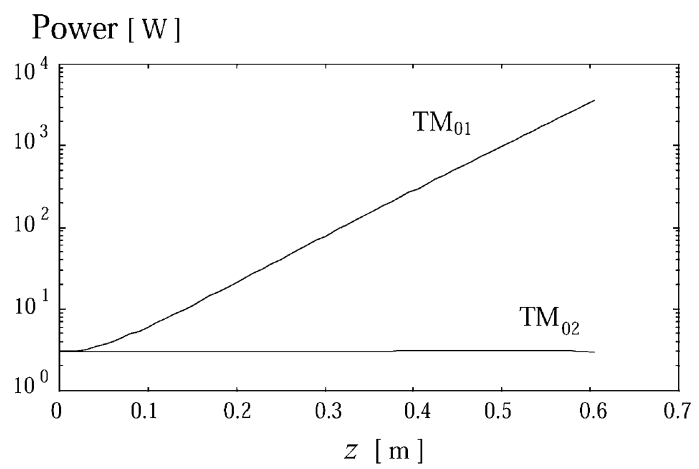

Fig. 10. Calculated output power of $\mathrm{TM}_{01}$ and $\mathrm{TM}_{02}$ modes as a function of

discharge chamber and the acceleration unit was located immediately after the collimator to achieve a gas pressure gradient and to optimize beam current. The acceleration unit was driven by a $40 \mathrm{kV}, 125$-ns voltage pulse produced by a cable Blumlein. The beam acceleration experiments showed that careful adjustment of the trigger system could ensure successful synchronization between the beam propagation and the application of the acceleration voltage. A 40-kV, 100-A electron beam pulse was measured at a distance of $120 \mathrm{~mm}$ from the acceleration gap without a magnetic guiding field, as shown in Fig. 9. However, in Fig. 9, some beam loading effect is evident where it can be seen that as the beam current increases the post acceleration voltage decreases and the flat top of the voltage signal becomes shorter. During the experiments, the beam loading effect was mitigated by reducing the internal impedance of the cable pulser from 50 to $14 \Omega$. It is possible to further reduce the beam loading effect by continuing to decrease the internal impedance of the cable Blumlein or by using an alternative lower impedance pulse forming line.

\section{SimULATIONS}

To complement the experimental investigations of the Cherenkov maser, a three-dimensional numerical-simulation code was developed. The code integrates the Lorentz equations of motion for the electrons self-consistently with Maxwell's wave equation for a single $\mathrm{TM}_{0 n}$ waveguide mode in the steady-state limit where the relative slippage of the radiation with respect to the electron beam is neglected [12], [13]. It includes the effect of resonant space-charge forces and electron velocity spread. Fig. 10 shows a graph of the predicted output power from the Cherenkov maser as a function of interaction 


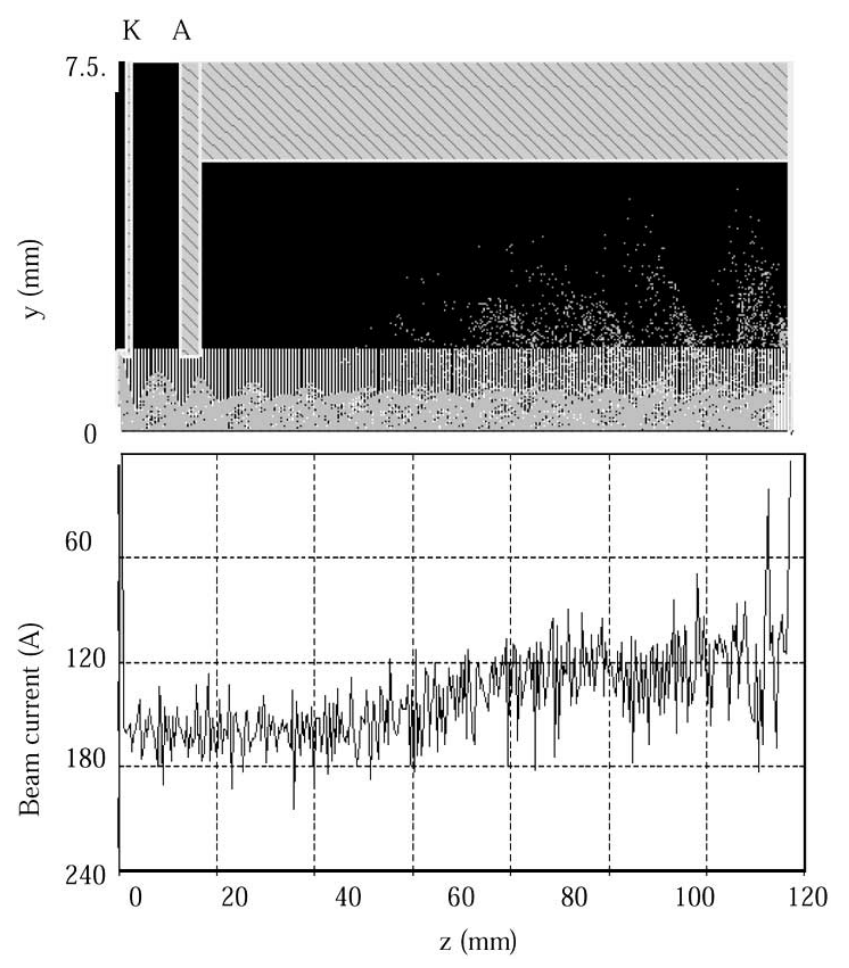

Fig. 11. Simulated beam profile and current variation during propagation across the acceleration gap and further along the beam channel with an ion background of density $6 \times 10^{12} \mathrm{~cm}^{-3}$ (K-cathode; A-anode; $\mathrm{z}$-beam channel axis; vertical dense lines-plasma zone; and cloud-electrons). Beam parameters: $200 \mathrm{~V}$ and $200 \mathrm{~A}$. Acceleration voltage: $40 \mathrm{kV}$ and $125 \mathrm{~ns}$. Acceleration gap: $5 \mathrm{~mm}$.

length for the $\mathrm{TM}_{01}$ and $\mathrm{TM}_{02}$ modes when the beam energy is $75 \mathrm{keV}$ and current is $10 \mathrm{~A}$. Only these two modes lie within the frequency range of the radiation emitted by the PS discharge. The microwave power at $z=0$ was assumed to be $3 \mathrm{~W}$ for both modes. The electron beam was assumed to be a perfectly collimated solid beam, with an axial velocity spread $\left(\Delta v_{z} / v_{z}\right)$ of $3 \%$, and a radius of $1.5 \mathrm{~mm}$. It should be stressed that there exists a large difference in the lateral and longitudinal energy spread. The lateral energy spread is large while the longitudinal energy spread is very small. It can be seen from Fig. 11 that the $\mathrm{TM}_{01}$ mode at $\sim 21 \mathrm{GHz}$ is amplified strongly, attaining a power of $\sim 3.4 \mathrm{~kW}$ at $z=60 \mathrm{~cm}$, whereas the power in the $\mathrm{TM}_{02}$ mode at $\sim 55 \mathrm{GHz}$ remains around its initial level. These simulations support the interpretation of the experimental results as microwave amplification via a Cherenkov interaction between the high-quality electron beam and the $\mathrm{TM}_{01}$ mode of the dielectric-lined waveguide.

Beam propagation across the acceleration gap and further along the beam channel was simulated using the electromagnetic particle-in-cell code MAGIC. This is a finite-difference, time-domain code for simulating plasma processes and those processes, which involve interactions between space charge and electromagnetic fields. The code is based on Maxwell's equations, the Lorentz force equation, and the continuity equation. Beginning from a specified initial state, the code simulates a physical process as it evolves in time. The full set of Maxwell's time-dependent equations is solved to obtain the electromagnetic fields. Similarly, the complete Lorentz force equation is solved to obtain relativistic particle trajectories, and the conti- nuity equation is solved to provide current and charge densities for Maxwell's equations. The simulations show that a $200-\mathrm{V}$, 200-A beam in a PS CP phase will propagate across a $40-\mathrm{kV}$ post-acceleration gap of 5-mm separation in a ion background of certain densities. About $10 \%$ and $70 \%$ of the beam would propagate across the gap when the ion densities are $1 \times 10^{12} \mathrm{~cm}^{-3}$ and $6 \times 10^{12} \mathrm{~cm}^{-3}$, respectively. Fig. 11 shows a simulated beam profile and current variation during its propagation across the acceleration gap in an ion background of density $6 \times 10^{12} \mathrm{~cm}^{-3}$. It can be seen that for an initial 200-A beam propagating across the acceleration gap and further along the drift tube, a 130-A beam can be obtained at a distance $120 \mathrm{~mm}$ away from the acceleration gap. In the experiment, a $100 \mathrm{~A}$ beam was measured at a distance about $120 \mathrm{~mm}$ away from the acceleration gap. The simulation also shows that with ion background, the shapes of both the cathode and anode of the acceleration gap have little effect on beam propagation.

\section{DISCUSSION AND CONCLUSION}

We have presented measurements of coherent electromagnetic radiation generation in a free-electron maser using an electron beam from a PS discharge. The microwave radiation was generated by Cherenkov amplification of the broadband emission from the PS discharge. A background signal level of around $100 \mathrm{~W}$ was measured in the frequency range $20-50 \mathrm{GHz}$ with a percentage of $(2.7 \pm 0.6) \%$ in the frequency range 25.5-28.6 $\mathrm{GHz}$, when the dielectric lining was removed from the maser. The frequency of the microwave output after the Cherenkov maser interaction was measured to be mainly around $25.5 \mathrm{GHz}$ and the dominating mode was identified as being $\mathrm{TM}_{01}$. The duration of the microwave pulse was approximately $80 \mathrm{~ns}$, with a peak power of around $2 \pm 0.2 \mathrm{~kW}$. The gain of this amplifier was measured as $29 \pm 3 \mathrm{~dB}$. The microwave output was found to be insensitive to the magnitude of the applied magnetic field, which was varied from 0.13 to $0.26 \mathrm{~T}$. The observed frequency, $25.5 \mathrm{GHz}$, was found to be slightly higher than that predicted by the resonance condition for the above beam parameters and the cold alumina-lined waveguide $(21 \mathrm{GHz})$. This discrepancy could be explained as the effect of either the charging of the dielectric liner or the beam loading effect on the transverse structure of the TM waveguide mode. Therefore, the two microwave pulses, that came from the two parts of the beam originating in the PS, had different frequencies and power amplifications in the Cherenkov interaction. Both the simulation and experiment show that the first microwave pulse of frequency of 25.5-28.6 $\mathrm{GHz}$ was amplified in the Cherenkov interaction with a gain of $29 \pm 3 \mathrm{~dB}$.

A 100-A, 40-kV electron beam pulse was measured at a distance of $120 \mathrm{~mm}$ from the acceleration gap without a magnetic guiding field. Comparing this with the simulation implies that a favorable ion background exists along the beam channel and the acceleration gap. The ion background can be formed from the background gas ionization by the initial high energy HCP beam during the PS discharge, which also expels electrons in the ionized gas media. In summary, the beam in the PS conductive phase was successfully accelerated from about $200 \mathrm{~V}$ to more than $40 \mathrm{kV}$. 
The future aims of this experiment are to improve the performance of the PS discharge with respect to microwave power, pulse repetition frequency, triggering accuracy while operating at high pulse-repetition frequencies, further reduction of the beam loading effect in the post acceleration unit and investigation of the transportation of this novel electron beam in the low-pressure gas-filled interaction region. It is clear that much higher output powers can be obtained from the Cherenkov maser amplifier by using an input signal rather than using the microwave output of the PS discharge. The ultimate goal of the research is to produce electron beam pulses, which offer very favorable comparison of their brightness with the very brightest available photocathode electron sources and have higher current densities, but at a small fraction of the cost of these systems. This work could potentially lead to a whole series of novel microwave sources and amplifiers employing a PS-based high-density high-brightness electron beam.

\section{ACKNOWLEDGMENT}

The authors would like to thank the high-power RF Faraday partnership for the provision of the MAGIC code and L. Ludeking of Mission Research Corporation for his helpful instruction in the use of MAGIC.

\section{REFERENCES}

[1] J. Christiansen and C. Schultheiss, "Production of high current particle beams by low pressure spark discharge," Z. Phys., vol. A290, pp. 35-39, 1979.

[2] M. A. Gunderson and G. Schaefer, "Physics and applications of pseudosparks," in NATO ASI, ser. B. New York: Plenum, 1990.

[3] K. Frank and J. Christiansen, "The fundamentals of the pseudospark and its applications," IEEE Trans. Plasma Sci., vol. 17, pp. 748-753, Oct. 1989.

[4] H. Yin, W. He, A. W. Cross, A. D. R. Phelps, and K. Ronald, "Single-gap pseudospark discharge experiments," J. Appl. Phys., vol. 90, pp. 3212-3218, Oct. 2001.

[5] H. Yin, A. D. R. Phelps, W. He, G. R. M. Robb, K. Ronald, P. Aitken, B. W. J. McNeil, A. W. Cross, and C. G. Whyte, "A pseudospark cathode Cherenkov maser: Theory and experiment," Nucl. Instrum. Meth. Phys. Res. A, vol. 407, pp. 175-180, 1998.

[6] H. Yin, W. He, G.R.M. Robb, A.D.R. Phelps, K. Ronald, and A. W. Cross, "Coherent microwave generation from a pseudospark cathode Cherenkov maser," Phys. Rev. Spec. Topics-Accelerat. Beams, vol. 2, pp. 1-5, 1999 .

[7] H. Yin, G. R. M. Robb, W. He, A. D. R. Phelps, A. W. Cross, and K. Ronald, "Pseudospark-based electron beam and Cherenkov maser experiments," Phys. Plasmas, vol. 7, pp. 5195-5204, Dec. 2000.

[8] H. Yin, A. W. Cross, A. D. R. Phelps, D. Zhu, W. He, and K. Ronald, "Propagation and post-acceleration of a pseudospark-sourced electron beam," J. Appl. Phys., vol. 91, pp. 5419-5422, Apr. 2002.

[9] R. Liou, H. Figueroa, A. H. McCurdy, G. Kirkman-Amemiya, R. J. Temkin, H. Fetterman, and M. A. Gundersen, "Emission of microwave and millimeter wavelength radiation during hollow cathode discharge operation of the back lighted thyratron," Appl. Phys. Lett., vol. 61, p. $2779,1992$.

[10] K. Ramaswamy, W. W. Destler, and J. Rodgers, "Microwave generation in a high voltage triggered pseudospark discharge experiment," J. Appl. Phys., vol. 83, pp. 3514-3520, 1998.

[11] E. Garate, R. Cook, P. Heim, R. Layman, and J. E. Walsh, "Cherenkov maser operation at lower-mm wavelengths," J. Appl. Phys., vol. 58, p. 627, 1985

[12] W. Peter, E. Garate, W. Main, and A. Fisher, "High-gain X-band dielectric Cherenkov maser," Phys. Rev. Lett., vol. 65, pp. 2989-2992, 1990.

[13] H. P. Freund, "Nonlinear analysis of high-power Cherenkov masers," Phys. Rev. Lett., vol. 65, pp. 2993-2996, 1990.

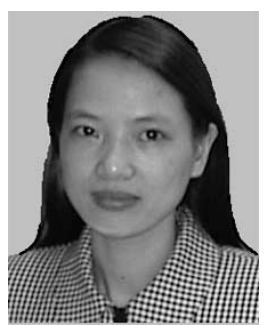

Huabi Yin received the B.Eng. degree from Huazhong University of Science and Technology, Wuhan, China, in 1983, the M.Sc. degree from the Graduate School of the China Academy of Engineering Physics, Chengdu, in 1988, and the Ph.D. degree in physics from the Department of Physics, University of Strathclyde, Glasgow, U.K., in 1998.

She is currently a Research Fellow with the Physics Department, University of Strathclyde. Her research interests include the area of pulsed-power technology, pseudospark discharges, intense electron beam production, and microwave sources based on beam-wave instabilities.

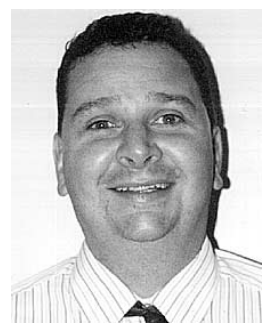

Adrian W. Cross received the B.Sc. degree (with honors) in physics and the Ph.D. degree from the University of Strathclyde, Glasgow, U.K., in 1989 and 1993, respectively.

He joined the Atoms, Beams, and Plasmas Group, Strathclyde University, in 1993 as a Research Fellow then as a Lecturer in 2000. He is currently a Senior Lecturer with the Department of Physics, Strathclyde University. He has been involved in various aspects of research on gyrotrons, cyclotron autoresonance masers, free electron lasers, superradiant sources, and plasma applications. More recently, he has primarily been concerned with research on radiation sources for use in accelerators and pseudospark physics.

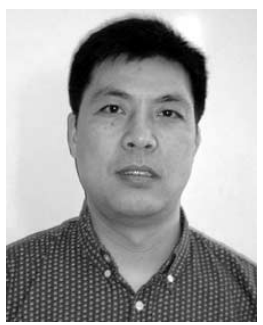

Wenlong He received the B.Sc. degree in physics in 1983 from Suchow University, Gangsu, China, the M.Sc. degree in accelerator physics from the China Academy of Engineering Physics, Chengdu, in 1988, and the Ph.D. degree in relativistic electron beams and masers from the Department of Physics, University of Strathclyde, Glasgow, U.K., in 1995.

He is currently a Senior Research Fellow with the Physics Department, University of Strathclyde. His main research interests include relativistic electron beams, CARMs, FELs, and gyro-TWTs.

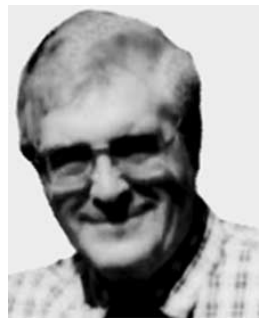

Alan D. R. Phelps was born in 1944 in the U.K. He received the B.A. degree (with honors) in physics and the M.A. degree from Cambridge University, Cambridge, U.K., in 1966 and 1970, respectively, and the D.Phil. degree for plasma research from Oxford University, Oxford, U.K. in 1976.

He founded a research group at Strathclyde University, Glasgow, U.K., in 1978, and became a full Professor in 1993. He was the Head of the Physics Department, Strathclyde University, from 1998 to 2001. His research interests include high-power free-electron radiation sources and plasmas.

Dr. Phelps is a Fellow of the Institute of Physics and of the Royal Society of Edinburgh.

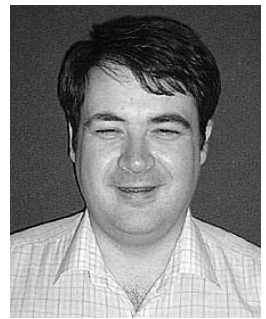

Kelvin Ronald was born in Glasgow, Scotland, U.K He received the B.Sc. degree (with honors) in physics and the Ph.D. degree in physics from the University of Strathclyde, Glasgow, in 1992 and 1997, respectively.

He is currently a Senior Research Fellow with the Physics Department, University of Strathclyde. 